Supplement of Geosci. Model Dev. Discuss., 8, 10455-10538, 2015

http://www.geosci-model-dev-discuss.net/8/10455/2015/

doi:10.5194/gmdd-8-10455-2015-supplement

(C) Author(s) 2015. CC Attribution 3.0 License.

(c) (i)

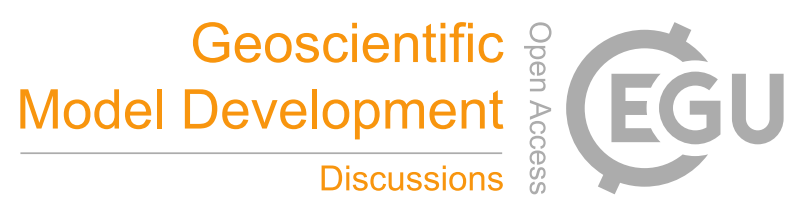

Supplement of

\title{
Development studies towards an 11-year global gridded aerosol optical thickness reanalysis for climate and applied applications
}

\section{P. Lynch et al.}

Correspondence to: P. Lynch (peng.lynch.ctr@nrlmry.navy.mil)

The copyright of individual parts of the supplement might differ from the CC-BY 3.0 licence. 


\section{Supplemental Material}

Table S1. Regional multipliers for smoke emission based on the FLAMBE 2-day-maximum MODIS fire hot spot signal and for dust erodibility based on Ginoux et al., 2001.

\begin{tabular}{|l|l|l|}
\hline region & Smoke emission & Dust erodibility \\
\hline Africa North & 0.8 & $\begin{array}{l}25 \mathrm{~N} \text { north } \times 0.4, \\
20 \mathrm{~N}-25 \mathrm{~N} \times 1.0, \\
20 \mathrm{~N} \text { south } \times 2.0\end{array}$ \\
\hline Africa South & 0.18 & 2.0 \\
\hline Australia & 1.0 & 0.5 \\
\hline Ctrl. America & 0.5 & 2.0 \\
\hline CONUS & 0.6 & 2.0 \\
\hline East Asia & 1.0 & 2.0 \\
\hline Eurasia Boreal & $30 \mathrm{E}$ east $\times 0.6$ west $\times 1.0$ & $\mathrm{~N} / \mathrm{A}$ \\
\hline EUR-Medit & 0.7 & 2.0 \\
\hline India & 1.0 & 2.0 \\
\hline Ins. SE Asia & 0.8 & $\mathrm{~N} / \mathrm{A}$ \\
\hline N. Am. Boreal & 0.7 & $\mathrm{~N} / \mathrm{A}$ \\
\hline Pen. SE Asia & 0.43 & $\mathrm{~N} / \mathrm{A}$ \\
\hline SW Asia & 1.0 & 1.5 \\
\hline South America & 0.3 & 0.5 \\
\hline Other & 1.0 & 2.0 \\
\hline
\end{tabular}


Table S2. Statistics of the reanlaysis total AOT at 550nm compared with AERONET L2 6-hr-average data for DJFMAM of 2003-2013. "Mean" and "Median" are the means and medians of the reanalysis regional AOTs. "AMean" and "AMedian" are the means and medians of the regional AERONET AOTs. Also shown are the bias and root mean square error ("RMSE") and the square of the Pearson correlation coefficient $\left(r^{2}\right)$. "Slope" and "Intercept" are the corresponding Theil-Sen linear regression parameters. " $N$ " is the total number of 6-hrly paired AERONET data in the region for validation.

\begin{tabular}{|c|c|c|c|c|c|c|c|c|c|c|}
\hline region & Mean & Median & AMean & AMedian & Bias & Rmse & $r^{2}$ & Slope & intercept & $\mathrm{N}$ \\
\hline Africa North & 0.36 & 0.24 & 0.40 & 0.27 & -0.04 & 0.25 & 0.68 & 0.88 & 0.01 & 42962 \\
\hline Africa South & 0.14 & 0.13 & 0.15 & 0.13 & -0.01 & 0.08 & 0.45 & 0.61 & 0.05 & 7934 \\
\hline Australia & 0.07 & 0.06 & 0.07 & 0.05 & 0.00 & 0.05 & 0.37 & 0.72 & 0.02 & 11103 \\
\hline Ctr. America & 0.16 & 0.12 & 0.18 & 0.12 & -0.02 & 0.11 & 0.71 & 0.64 & 0.04 & 12093 \\
\hline EAST CONUS & 0.12 & 0.11 & 0.11 & 0.09 & 0.01 & 0.06 & 0.65 & 0.86 & 0.03 & 39244 \\
\hline East Asia & 0.34 & 0.27 & 0.40 & 0.29 & -0.06 & 0.25 & 0.61 & 0.63 & 0.08 & 26993 \\
\hline Eurasian Boreal & 0.16 & 0.13 & 0.17 & 0.13 & -0.02 & 0.09 & 0.57 & 0.58 & 0.05 & 24149 \\
\hline EUR-Medit & 0.15 & 0.13 & 0.15 & 0.12 & 0.00 & 0.08 & 0.57 & 1.72 & -0.07 & 79008 \\
\hline Indian & 0.37 & 0.34 & 0.42 & 0.38 & -0.05 & 0.19 & 0.54 & 0.62 & 0.10 & 18770 \\
\hline Ins. SE Asia & 0.18 & 0.16 & 0.23 & 0.18 & -0.05 & 0.13 & 0.37 & 0.44 & 0.07 & 4448 \\
\hline N. Am. Boreal & 0.11 & 0.10 & 0.09 & 0.07 & 0.02 & 0.05 & 0.46 & 0.70 & 0.05 & 10460 \\
\hline ocean & 0.12 & 0.10 & 0.10 & 0.07 & 0.02 & 0.07 & 0.68 & 0.56 & 0.06 & 15645 \\
\hline Pen. SE Asia & 0.44 & 0.36 & 0.50 & 0.42 & -0.06 & 0.21 & 0.66 & 0.75 & 0.05 & 12146 \\
\hline S. America & 0.10 & 0.09 & 0.09 & 0.07 & 0.01 & 0.07 & 0.23 & 0.42 & 0.06 & 17563 \\
\hline SW. Asia & 0.38 & 0.32 & 0.35 & 0.29 & 0.02 & 0.20 & 0.54 & 0.84 & 0.07 & 10496 \\
\hline WEST CONUS & 0.10 & 0.10 & 0.08 & 0.06 & 0.03 & 0.06 & 0.30 & 0.68 & 0.05 & 32145 \\
\hline Globe & 0.20 & 0.13 & 0.21 & 0.12 & -0.01 & 0.14 & 0.71 & 0.94 & 0.01 & 365159 \\
\hline
\end{tabular}


Table S3. Same as Table S2, except for total AOT at 550nm during JJASON of 2003-2013.

\begin{tabular}{|c|c|c|c|c|c|c|c|c|c|c|}
\hline region & Mean & Median & AMean & AMedian & Bias & Rmse & $r^{2}$ & Slope & intercept & $\mathrm{N}$ \\
\hline Africa North & 0.32 & 0.27 & 0.34 & 0.27 & -0.02 & 0.20 & 0.57 & 0.81 & 0.05 & 39732 \\
\hline Africa South & 0.21 & 0.17 & 0.23 & 0.19 & -0.03 & 0.09 & 0.77 & 0.76 & 0.02 & 8312 \\
\hline Australia & 0.09 & 0.07 & 0.11 & 0.07 & -0.01 & 0.07 & 0.59 & 0.71 & 0.01 & 13889 \\
\hline Ctr. America & 0.18 & 0.14 & 0.20 & 0.15 & -0.02 & 0.10 & 0.59 & 0.67 & 0.04 & 9339 \\
\hline EAST CONUS & 0.16 & 0.13 & 0.18 & 0.13 & -0.01 & 0.09 & 0.70 & 0.91 & 0.01 & 49820 \\
\hline East Asia & 0.28 & 0.21 & 0.37 & 0.22 & -0.09 & 0.31 & 0.61 & 0.52 & 0.08 & 26487 \\
\hline Eurasian Boreal & 0.14 & 0.12 & 0.15 & 0.12 & -0.01 & 0.08 & 0.64 & 0.67 & 0.04 & 41112 \\
\hline EUR-Medit & 0.17 & 0.14 & 0.17 & 0.14 & -0.01 & 0.08 & 0.58 & 1.00 & 0.00 & 109530 \\
\hline Indian & 0.45 & 0.41 & 0.48 & 0.43 & -0.03 & 0.24 & 0.55 & 0.65 & 0.12 & 11248 \\
\hline Ins. SE Asia & 0.27 & 0.22 & 0.37 & 0.26 & -0.10 & 0.29 & 0.44 & 0.49 & 0.08 & 3906 \\
\hline N. Am. Boreal & 0.11 & 0.09 & 0.09 & 0.06 & 0.01 & 0.08 & 0.64 & 0.80 & 0.03 & 19900 \\
\hline ocean & 0.12 & 0.09 & 0.10 & 0.07 & 0.02 & 0.06 & 0.70 & 0.59 & 0.05 & 16470 \\
\hline Pen. SE Asia & 0.28 & 0.22 & 0.33 & 0.22 & -0.04 & 0.18 & 0.70 & 0.59 & 0.08 & 5468 \\
\hline S. America & 0.20 & 0.11 & 0.22 & 0.12 & -0.02 & 0.14 & 0.82 & 0.71 & 0.03 & 23262 \\
\hline SW. Asia & 0.45 & 0.40 & 0.41 & 0.36 & 0.04 & 0.16 & 0.64 & 0.86 & 0.09 & 14128 \\
\hline WEST CONUS & 0.11 & 0.10 & 0.09 & 0.07 & 0.02 & 0.07 & 0.39 & 0.76 & 0.04 & 43166 \\
\hline Globe & 0.19 & 0.14 & 0.20 & 0.13 & -0.01 & 0.14 & 0.67 & 1.05 & 0.00 & 435769 \\
\hline
\end{tabular}


Table S4. Same as Table S2, except for fine mode AOT at 550nm during DJFMAM of 2003-2013.

\begin{tabular}{|l|r|r|r|r|r|r|r|r|r|r|}
\hline region & \multicolumn{1}{|l}{ Mean } & \multicolumn{1}{l|}{ Median } & \multicolumn{1}{l|}{ AMean } & \multicolumn{1}{l|}{ AMedian } & \multicolumn{1}{l|}{ Bias } & \multicolumn{1}{l|}{ Rmse } & \multicolumn{1}{l|}{$r^{2}$} & \multicolumn{1}{l}{ Slope } & intercept & $\mathrm{N}$ \\
\hline Africa North & 0.11 & 0.07 & 0.11 & 0.07 & 0.00 & 0.10 & 0.46 & 0.99 & 0.01 & 42962 \\
\hline Africa South & 0.11 & 0.09 & 0.08 & 0.06 & 0.03 & 0.06 & 0.44 & 0.87 & 0.03 & 7934 \\
\hline Australia & 0.04 & 0.03 & 0.03 & 0.02 & 0.01 & 0.03 & 0.46 & 0.99 & 0.00 & 11103 \\
\hline Ctr. America & 0.10 & 0.06 & 0.10 & 0.05 & 0.00 & 0.09 & 0.74 & 0.86 & 0.01 & 12093 \\
\hline EAST CONUS & 0.08 & 0.06 & 0.07 & 0.05 & 0.02 & 0.04 & 0.65 & 1.11 & 0.01 & 39244 \\
\hline East Asia & 0.23 & 0.16 & 0.24 & 0.14 & -0.02 & 0.17 & 0.62 & 0.76 & 0.03 & 26993 \\
\hline Eurasian Boreal & 0.10 & 0.08 & 0.10 & 0.07 & -0.01 & 0.06 & 0.60 & 0.71 & 0.02 & 24149 \\
\hline EUR-Medit & 0.08 & 0.07 & 0.08 & 0.06 & 0.00 & 0.05 & 0.48 & 1.29 & -0.01 & 79008 \\
\hline Indian & 0.24 & 0.21 & 0.22 & 0.17 & 0.02 & 0.15 & 0.49 & 0.72 & 0.08 & 18770 \\
\hline Ins. SE Asia & 0.14 & 0.12 & 0.14 & 0.10 & 0.01 & 0.10 & 0.36 & 0.62 & 0.05 & 4448 \\
\hline N. Am. Boreal & 0.06 & 0.05 & 0.05 & 0.04 & 0.01 & 0.03 & 0.54 & 0.80 & 0.02 & 10460 \\
\hline Ocean & 0.05 & 0.04 & 0.04 & 0.02 & 0.01 & 0.04 & 0.72 & 0.91 & 0.01 & 15645 \\
\hline Pen. SE Asia & 0.40 & 0.32 & 0.37 & 0.28 & 0.03 & 0.19 & 0.67 & 0.85 & 0.07 & 12146 \\
\hline S. America & 0.05 & 0.05 & 0.05 & 0.04 & 0.01 & 0.04 & 0.38 & 0.69 & 0.02 & 17563 \\
\hline SW. Asia & 0.13 & 0.11 & 0.10 & 0.09 & 0.03 & 0.07 & 0.26 & 0.81 & 0.04 & 10496 \\
\hline WEST CONUS & 0.05 & 0.05 & 0.04 & 0.03 & 0.02 & 0.03 & 0.28 & 0.82 & 0.02 & 32145 \\
\hline Globe & 0.11 & 0.07 & 0.11 & 0.06 & 0.01 & 0.09 & 0.67 & 1.23 & 0.00 & 365159 \\
\hline
\end{tabular}


Table S5. Same as Table S2, except for fine mode AOT at 550nm during JJASON of 2003-2013.

\begin{tabular}{|c|c|c|c|c|c|c|c|c|c|c|}
\hline region & Mean & Median & AMean & AMedian & Bias & Rmse & $r^{2}$ & Slope & intercept & $\mathrm{N}$ \\
\hline Africa North & 0.12 & 0.10 & 0.08 & 0.07 & 0.04 & 0.09 & 0.29 & 1.10 & 0.02 & 39732 \\
\hline Africa South & 0.16 & 0.13 & 0.16 & 0.11 & 0.01 & 0.07 & 0.77 & 0.87 & 0.02 & 8312 \\
\hline Australia & 0.05 & 0.03 & 0.06 & 0.03 & -0.01 & 0.04 & 0.62 & 0.84 & 0.00 & 13889 \\
\hline Ctr. America & 0.07 & 0.06 & 0.07 & 0.05 & 0.00 & 0.06 & 0.44 & 0.77 & 0.01 & 9339 \\
\hline EAST CONUS & 0.13 & 0.10 & 0.12 & 0.08 & 0.01 & 0.07 & 0.70 & 1.12 & 0.00 & 49820 \\
\hline East Asia & 0.20 & 0.13 & 0.25 & 0.12 & -0.05 & 0.24 & 0.65 & 0.62 & 0.04 & 26487 \\
\hline Eurasian Boreal & 0.10 & 0.08 & 0.10 & 0.07 & 0.00 & 0.06 & 0.68 & 0.78 & 0.02 & 41112 \\
\hline EUR-Medit & 0.10 & 0.09 & 0.10 & 0.08 & 0.01 & 0.06 & 0.51 & 1.15 & 0.00 & 109530 \\
\hline Indian & 0.30 & 0.26 & 0.25 & 0.18 & 0.04 & 0.18 & 0.48 & 0.69 & 0.11 & 11248 \\
\hline Ins. SE Asia & 0.23 & 0.18 & 0.26 & 0.15 & -0.03 & 0.23 & 0.44 & 0.59 & 0.06 & 3906 \\
\hline N. Am. Boreal & 0.08 & 0.06 & 0.06 & 0.04 & 0.01 & 0.07 & 0.64 & 0.99 & 0.01 & 19900 \\
\hline ocean & 0.05 & 0.04 & 0.04 & 0.02 & 0.01 & 0.04 & 0.72 & 0.81 & 0.02 & 16470 \\
\hline Pen. SE Asia & 0.24 & 0.17 & 0.22 & 0.12 & 0.02 & 0.14 & 0.74 & 0.75 & 0.07 & 5468 \\
\hline S. America & 0.16 & 0.07 & 0.15 & 0.07 & 0.01 & 0.12 & 0.84 & 0.95 & 0.01 & 23262 \\
\hline SW. Asia & 0.18 & 0.17 & 0.15 & 0.13 & 0.03 & 0.07 & 0.34 & 0.65 & 0.08 & 14128 \\
\hline WEST CONUS & 0.07 & 0.06 & 0.06 & 0.04 & 0.02 & 0.06 & 0.44 & 1.04 & 0.02 & 43166 \\
\hline Globe & 0.12 & 0.08 & 0.11 & 0.07 & 0.01 & 0.10 & 0.65 & 1.33 & -0.01 & 435769 \\
\hline
\end{tabular}


Table S6. Same as Table S2, except for coarse mode AOT at 550nm during DJFMAM of 2003-2013.

\begin{tabular}{|c|c|c|c|c|c|c|c|c|c|c|}
\hline region & Mean & Median & AMean & AMedian & Bias & Rmse & $r^{2}$ & Slope & intercept & $\mathrm{N}$ \\
\hline Africa North & 0.25 & 0.14 & 0.29 & 0.18 & -0.05 & 0.22 & 0.60 & 0.77 & 0.01 & 42962 \\
\hline Africa South & 0.04 & 0.03 & 0.07 & 0.06 & -0.04 & 0.06 & 0.38 & 0.31 & 0.01 & 7934 \\
\hline Australia & 0.04 & 0.03 & 0.04 & 0.03 & 0.00 & 0.04 & 0.15 & 0.40 & 0.02 & 11103 \\
\hline Ctr. America & 0.06 & 0.05 & 0.08 & 0.07 & -0.02 & 0.06 & 0.26 & 0.25 & 0.04 & 12093 \\
\hline EAST CONUS & 0.04 & 0.04 & 0.05 & 0.03 & 0.00 & 0.03 & 0.29 & 0.45 & 0.02 & 39244 \\
\hline East Asia & 0.12 & 0.09 & 0.16 & 0.12 & -0.05 & 0.16 & 0.32 & 0.42 & 0.04 & 26993 \\
\hline Eurasian Boreal & 0.06 & 0.05 & 0.07 & 0.05 & -0.01 & 0.05 & 0.25 & 0.38 & 0.03 & 24149 \\
\hline EUR-Medit & 0.07 & 0.05 & 0.07 & 0.05 & 0.00 & 0.05 & 0.60 & 2.15 & -0.05 & 79008 \\
\hline Indian & 0.13 & 0.10 & 0.20 & 0.15 & -0.07 & 0.13 & 0.53 & 0.53 & 0.01 & 18770 \\
\hline Ins. SE Asia & 0.04 & 0.03 & 0.09 & 0.08 & -0.05 & 0.07 & 0.02 & 0.08 & 0.03 & 4448 \\
\hline N. Am. Boreal & 0.05 & 0.05 & 0.04 & 0.03 & 0.01 & 0.03 & 0.21 & 0.50 & 0.03 & 10460 \\
\hline ocean & 0.07 & 0.06 & 0.06 & 0.05 & 0.01 & 0.05 & 0.53 & 0.25 & 0.05 & 15645 \\
\hline Pen. SE Asia & 0.04 & 0.03 & 0.13 & 0.12 & -0.09 & 0.11 & 0.15 & 0.15 & 0.01 & 12146 \\
\hline S. America & 0.05 & 0.04 & 0.04 & 0.03 & 0.01 & 0.05 & 0.03 & 0.18 & 0.03 & 17563 \\
\hline SW. Asia & 0.25 & 0.19 & 0.26 & 0.19 & -0.01 & 0.18 & 0.51 & 0.77 & 0.04 & 10496 \\
\hline WEST CONUS & 0.05 & 0.05 & 0.04 & 0.03 & 0.01 & 0.04 & 0.18 & 0.51 & 0.03 & 32145 \\
\hline Globe & 0.09 & 0.05 & 0.11 & 0.06 & -0.02 & 0.11 & 0.62 & 0.85 & 0.01 & 365159 \\
\hline
\end{tabular}


Table S7. Same as Table S2, except for coarse mode AOT at 550nm during JJASON of 2003-2013.

\begin{tabular}{|c|c|c|c|c|c|c|c|c|c|c|}
\hline region & Mean & Median & AMean & AMedian & Bias & Rmse & $r^{2}$ & Slope & intercept & $\mathrm{N}$ \\
\hline Africa North & 0.21 & 0.15 & 0.26 & 0.19 & -0.05 & 0.19 & 0.52 & 0.70 & 0.02 & 39732 \\
\hline Africa South & 0.04 & 0.04 & 0.08 & 0.07 & -0.04 & 0.05 & 0.42 & 0.41 & 0.01 & 8312 \\
\hline Australia & 0.04 & 0.03 & 0.05 & 0.03 & -0.01 & 0.04 & 0.21 & 0.45 & 0.01 & 13889 \\
\hline Ctr. America & 0.10 & 0.07 & 0.12 & 0.08 & -0.02 & 0.07 & 0.68 & 0.63 & 0.02 & 9339 \\
\hline EAST CONUS & 0.03 & 0.03 & 0.05 & 0.04 & -0.02 & 0.04 & 0.26 & 0.42 & 0.01 & 49820 \\
\hline East Asia & 0.08 & 0.06 & 0.11 & 0.09 & -0.04 & 0.12 & 0.11 & 0.26 & 0.04 & 26487 \\
\hline Eurasian Boreal & 0.04 & 0.04 & 0.06 & 0.05 & -0.02 & 0.04 & 0.20 & 0.44 & 0.01 & 41112 \\
\hline EUR-Medit & 0.06 & 0.05 & 0.07 & 0.05 & -0.01 & 0.05 & 0.58 & 0.89 & 0.00 & 109530 \\
\hline Indian & 0.15 & 0.10 & 0.22 & 0.16 & -0.07 & 0.16 & 0.60 & 0.61 & 0.00 & 11248 \\
\hline Ins. SE Asia & 0.04 & 0.04 & 0.11 & 0.10 & -0.07 & 0.11 & 0.04 & 0.14 & 0.02 & 3906 \\
\hline N. Am. Boreal & 0.03 & 0.03 & 0.03 & 0.02 & 0.00 & 0.03 & 0.10 & 0.34 & 0.02 & 19900 \\
\hline ocean & 0.06 & 0.05 & 0.05 & 0.04 & 0.01 & 0.05 & 0.58 & 0.33 & 0.04 & 16470 \\
\hline Pen. SE Asia & 0.04 & 0.03 & 0.11 & 0.10 & -0.07 & 0.10 & 0.08 & 0.08 & 0.02 & 5468 \\
\hline S. America & 0.04 & 0.03 & 0.07 & 0.05 & -0.03 & 0.07 & 0.03 & 0.12 & 0.02 & 23262 \\
\hline SW. Asia & 0.27 & 0.22 & 0.26 & 0.21 & 0.01 & 0.14 & 0.62 & 0.93 & 0.02 & 14128 \\
\hline WEST CONUS & 0.04 & 0.04 & 0.04 & 0.03 & 0.00 & 0.03 & 0.03 & 0.31 & 0.03 & 43166 \\
\hline Globe & 0.07 & 0.04 & 0.09 & 0.05 & -0.02 & 0.08 & 0.61 & 0.87 & 0.00 & 435769 \\
\hline
\end{tabular}

